\title{
Evaluation of in vitro plant regeneration efficiency in Siberian wheat cultivars
}

\author{
D.N. Miroshnichenko ${ }^{1 *}$, A.A. Klementyeva ${ }^{1}$, E.A. Salina² ${ }^{2}$ S.V. Dolgov ${ }^{1}$ \\ ${ }^{1}$ Branch of Shemyakin and Ovchinnikov Institute of Bioorganic Chemistry RAS, Pushchino, Moscow Region, Russia \\ 2 Institute of Cytology and Genetics, SB RAS, Novosibirsk, Russia
}

DOI 10.18699/ICG-PlantGen2019-40

(c) Autors, 2019

* e-mail: miroshnichenko@bibch.ru

\begin{abstract}
The regeneration ability of three Siberian bread wheat cultivars, 'Novosibirskaya-40', 'Novosibirskaya-61' and 'Velut', has been studied. Embryogenic callus formation and shoot regeneration were successfully achieved from the isolated immature zygotic embryos using a two-step regeneration protocol. Embryogenic calli were induced by culturing immature embryos on a medium supplemented with 2,4-dichlorophenoxyacetic acid $(2,4-D)$. During the first step of culture, all the cultivars were able to produce embryogenic calli with a high frequency varying from $87.1 \%$ ('Novosibirskaya-40') to $95.5 \%$ ('Novosibirskaya-61'). Upon withdrawal from auxin, differentiation of embryogenic structures into green plantlets was achieved. The ability to regenerate plants in vitro differed among the wheat cultivars tested, the highest was observed for 'Velut' (13.7 plantlets per morphogenic explant) and the lowest, for 'Novosibirskaya-40' (5.7 plantlets per morphogenic explant).

Key words: Triticum aestivum L.; immature embryos; somatic embryogenesis; plant regeneration.
\end{abstract}

\section{Introduction}

The complementing of the traditional breeding approaches with the modern biotechnologies opens the possibility to enhance genetic variation in wheat. Different new breeding technologies (NBTs) such as haploid production, genetic engineering, or plant genome editing strongly depend on the availability of cultivars demonstrating efficient in vitro plants regeneration. In last decades, the application of NBTs to genetically improve the diversity of the wheat genome was repeatedly reported (Shrawat, Armstrong, 2018; Borisjuk et al., 2019). However, most of the researches were mostly carried out using 'model' cultivars, while the application of NBTs to high-quality local cultivars has a more practical perspective.

In wheat, plant regeneration can be achieved through embryogenesis or organogenesis induced by the culturing of isolated immature zygotic embryos on a medium supplemented with auxins (Delporte et al., 2014). Our previous results showed that the 4-week culture of immature tissues on a media containing 2,4-D (2,4-dichlorophenoxyacetic acid) under darkness with the subsequent plant differentiation under light on media without phytohormones could be good starting practice for testing various wheat cultivars and species for regeneration abilities (Alikina et al., 2016). In this study, Siberian cultivars of bread wheat adapted to local environments were investigated for their ability to induce embryogenic calli and produce plants in vitro.

\section{Materials and methods}

Three different samples of bread wheat (Triticum aestivum L.) - cv. 'Novosibirskaya-40' (winter), cv. 'Novosibirskaya-61' (spring) and line 'Velut' (spring) - were tested. The wheat samples differ in genomic composition. In contrast to cv 'Novosibirskaya-40' the other two wheat samples have translocation from wild species: cv. Novosibirskaya-61 - from Agropyron and line 'Velut' - from rye and Aegilops speltodies (Salina, unpublished). The donor plants growing and the immature embryos isolation were performed as described (Alikina et al., 2016). To ensure a sufficient number (not less than 60 in general) of embryogenic calli formed and used to regenerate plants, 115-135 initial explants for each variety were placed on the induction medium.

Callus induction media used in this study consisted of MS macro- and microelements and vitamins (Murashige and Skoog, 1962), 2 mg/L 2,4-D, 150 mg/L L-asparagine, $3 \%$ $(\mathrm{w} / \mathrm{v})$ sucrose, and were solidified with $7 \mathrm{~g} / \mathrm{L}$ agar, $\mathrm{pH} 5.8$. The frequencies of embryogenic callus formation from immature embryos were calculated after 1 month of culture. For plant regeneration, embryogenic calli produced by immature embryos were transferred into culture flasks on a hormonefree medium containing MS mineral salts and vitamins, $20 \mathrm{~g} / \mathrm{L}$ sucrose, and $7 \mathrm{~g} / \mathrm{L}$ agar. At the end of culturing, the number of plantlets regenerated from embryogenic calli was calculated.

\section{Results and discussion}

An important prerequisite for the production of transgenic or genome-edited wheat plants is the availability of a target tissue competent for plant regeneration. In wheat, the most popular explants routinely used to induce plant regeneration are immature zygotic tissues (Shrawat, Armstrong, 2018). For many cultivars of polyploid wheat, it is usually sufficient to use the supplement of 2-3 mg/l of 2,4-D to induce somatic embryogenesis from isolated immature zygotic embryos (Delporte et al., 2014). In some cases, certain germplasms display significant recalcitrance in vitro, so optimization is required to achieve an appreciable level of morphogenic response with regard to the choice of auxin type and its proper concentration (Miroshnichenko et al., 2016, 2017). In the present study, we have made an attempt to study the morphogenic efficiency of three local wheat cultivars, 'Novosibirskaya-40', 'Novosibirskaya-61' and 'Velut'. Since the in vitro response of zygotic tissues in wheat strictly depends on the genotype, this research was aimed to clarify which of the cultivars could be 
Table 1

In vitro culture response of immature embryo tissue of three Siberian wheat cultivars

\begin{tabular}{llll}
\hline Cultivar & $\begin{array}{l}\text { Callus induction*, } \\
(\%)\end{array}$ & $\begin{array}{l}\text { Embryogenic callus induction**, } \\
(\%)\end{array}$ & No. of regenerated shoots*** \\
\hline Novosibirskaya-40 & $94.0 \pm 5.5$ & $87.1 \pm 9.6$ & $5.7 \pm 0.9$ \\
Novosibirskaya-61 & $99.3 \pm 1.8$ & $95.5 \pm 5.6$ & $8.0 \pm 1.2$ \\
Velut & $97.4 \pm 2.5$ & $93.9 \pm 8.7$ & $13.7 \pm 1.9$ \\
\hline
\end{tabular}

* Percentage of initial explants producing callus

** Percentage of initial explants producing embryogenic callus

*** Number of regenerated plantlets per embryogenic callus

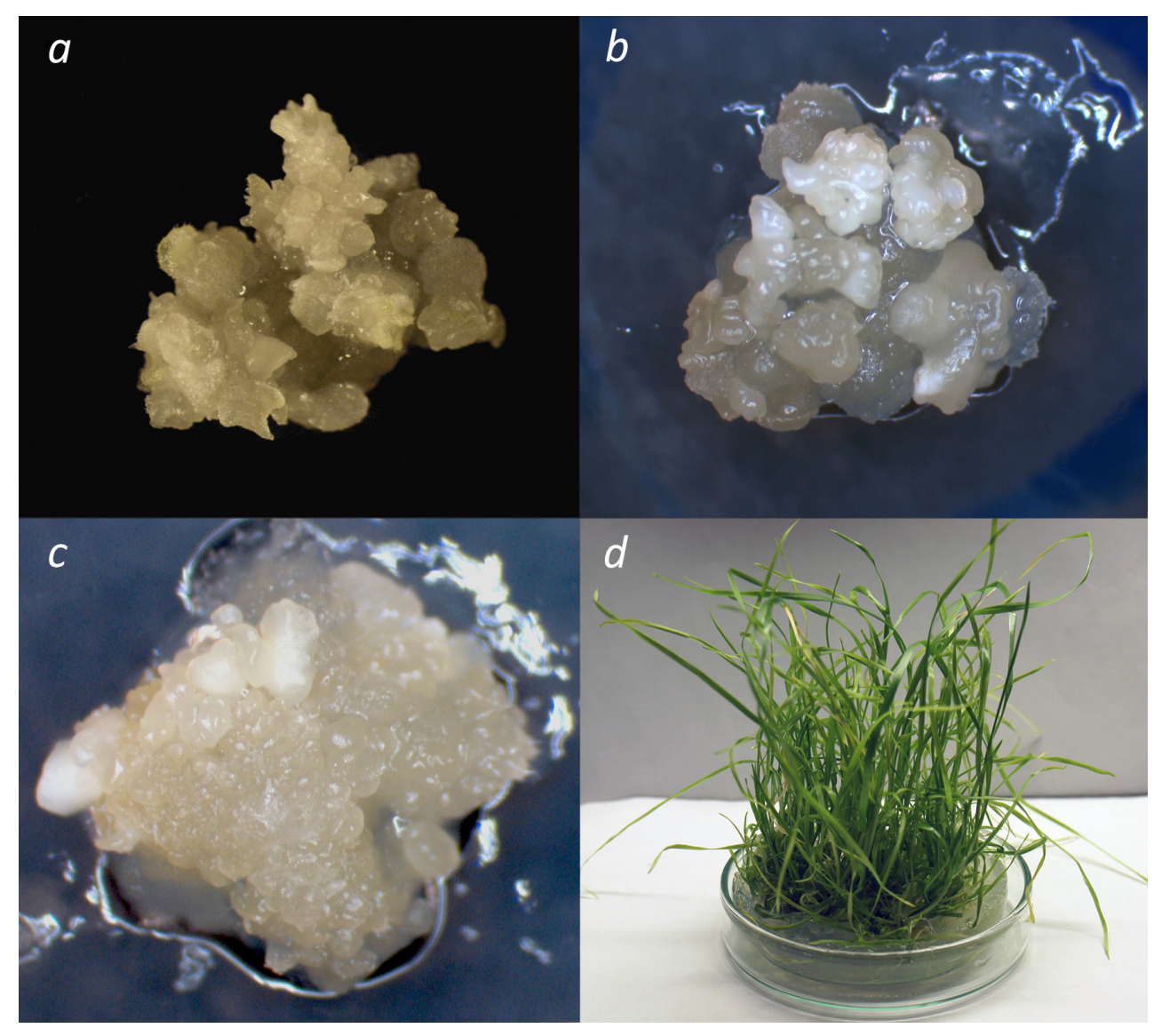

Figure 1. Embryogenic callus formation from immature embryo tissues of 'Novosibirskaya-61' (a), Novosibirskaya-40' (b), 'Velut' (c) on the medium supplemented with $2 \mathrm{mg} / \mathrm{L} \mathrm{2,4-D}$ after 4 weeks of culture, and regeneration of plants from embryogenic callus of 'Velut' $(d)$ after 30 days of culture on medium without growth regulators.

directly used for the NBT programs, and which require the further optimization of the in vitro morphogenesis protocol.

Within two weeks of cultivation, the formation of embryogenic callus surrounded by translucent non-embryogenic callus was observed in all cultivars. Taking into account the efficiency of callogenesis and embryogenesis (Table 1), no clear differences were found between cultivars. All the cultivars studied displayed a high ability to form embryogenic structures, varying from $87.1 \%$ ('Novosibirskaya-40') to $95.5 \%$ ('Novosibirskaya-61'). However, the pattern of the formation of the morphogenic structures differed in the cultivars studied.
'Novosibirskaya-61' and especially 'Novosibirskaya-40' explants formed mostly scattered embryogenic structures (Figure $1, a, b)$, while the entire explant surface of 'Velut' was usually covered with numerous embryogenic structures at the end of culture period on auxin-containing medium (see Figure 1,c).

The difference in the density of morphogenic structure formation significantly influenced the regeneration capacity of the cultivars. After transfer onto the medium lacking plant growth regulators, morphogenic explants of 'Velut' displayed high conversion into plantlets (Figure 1,d) - they reached the figure of 13.7 plantlets per explant (Table 1). In contrast, 
embryogenic callus of 'Novosibirskaya-40' and 'Novosibirskaya-61' generated only 5.7 and 8.0 plantlets, respectively. Despite the difference in the number of plants produced, none of the cultivars showed the formation of albino individuals among regenerated plants, a phenomenon previously reported for polyploid wheat germplasms belonging to T. kiharae, T. timopheevii, T. carthlicum and T. compactum (Alikina et al., 2016; Miroshnichenko et al., 2016).

Regenerated shoots of 'Novosibirskaya-40', 'Novosibirskaya-61' and 'Velut' with prominent roots were further transferred into the greenhouse. All plantlets that had been transferred to soil survived the greenhouse conditions and grew into morphologically normal and fertile plants.

\section{Conclusions}

The two-step protocol based on 2,4-D as the main exogenous inducer for the in vitro response of local Siberian cultivars of bread wheat can be successfully used to generate a sustainable supply of morphogenic material from immature embryos for the application in various NBT programs. All cultivars studied demonstrated an appreciable ability to produce embryogenic callus, which is the main target for delivering constructs encoding genome editing components or transgenic sequences. Some modifications of the protocol, however, should be recommended to control more efficiently the conversion of morphogenic structures into the whole plants in 'Novosibirskaya-40' and 'Novosibirskaya-61'. As an example, a combination of various plant growth regulators displaying distinctive biological activities (Miroshnichenko et al., 2017) could be simultaneously used for promoting a higher rate of morphogenesis in these wheat cultivars.

\section{References}

Alikina O., Chernobrovkina M., Dolgov S., Miroshnichenko D. Tissue culture efficiency of wheat species with different genomic formulas. Crop Breeding and Applied Biotechnology. 2016;16:307-314. DOI 10.1590/1984-70332016v16n4a46

Borisjuk N., Kishchenko O., Eliby S., Schramm C., Anderson P., Jatayev S., Kurishbayev A., Shavrukov Y. Genetic modification for wheat improvement: from transgenesis to genome editing. Biomed Res Int. 2019;2019:6216304. DOI 10.1155/2019/6216304.

Delporte F., Pretova A., du Jardin P., Watillon B. Morpho-histology and genotype dependence of in vitro morphogenesis in mature embryo cultures of wheat. Protoplasma. 2014;251(6):1455-1470. DOI 10.1007/s00709-014-0647-7.

Miroshnichenko D., Chaban I., Chernobrovkina M., Dolgov S. Protocol for efficient regulation of in vitro morphogenesis in einkorn (Triticum monococcum L.), a recalcitrant diploid wheat species. PLoS ONE. 2017;12(3):e0173533. DOI 10.1371/journal.pone.0173533.

Miroshnichenko D., Chernobrovkina M., Dolgov S. Somatic embryogenesis and plant regeneration from immature embryos of Triticum timopheevii Zhuk. and Triticum kiharae Dorof. et Migusch, wheat species with G genome. Plant Cell Tiss Organ Cult. 2016;125(3): 495-508. DOI 10.1007/s11240-016-0965-x.

Murashige T., Skoog F. A revised medium for rapid growth and bioassays with tobacco tissue cultures. Physiol Plant. 1962;15:473-497.

Shrawat A.K., Armstrong C.L. Development and application of genetic engineering for wheat improvement. Crit Rev Plant Sci. 2018; 37(5):335-421. DOI 10.1080/07352689.2018.1514718.

Conflict of interest. The authors declare no conflict of interest. 